\title{
Prognostic value of CC-chemokine receptor seven expression in patients with metastatic renal cell carcinoma treated with tyrosine kinase inhibitor
}

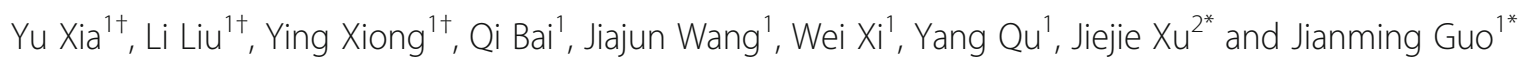

\begin{abstract}
Background: CC-chemokine receptor seven (CCR7), a G-protein coupled receptor normally facilitating immune cells lymphatic homing, has recently been identified on several cancer cells in promoting invasion and lymphatic specific metastasis by mimicking normal leukocytes. As tyrosine kinase inhibitors for metastatic renal cell carcinoma (mRCC) mostly emphasized on vascular inhibition, whether the CCR7 expressing tumor cells with potential lymphatic invasion function could have an impact on mRCC patient's drug response and survival, was unknown.

Methods: In this study, in a clinical aspect, we retrospectively investigated the prognostic and predictive impact of tumoral CCR7 expression in 110 mRCC patients treated with sunitinib and sorafenib, and its correlation with pre- or post-administration lymphatic involvement. Immunohistochemistry on tissue microarrays were conducted for CCR7 expression evaluation.

Results: Kaplan-Meier and univariate analyses suggested high tumoral CCR7 expression as an adverse prognosticator for mRCC patients' overall survival (OS), which was further confirmed in the multivariate analyses $(P=0.002, P=0.003$ for bootstrap). This molecule could be combined with Heng's risk model for better patient OS prediction. High tumoral CCR7 expression was also an independent dismal predictor for patients' progression free survival (PFS) $(P=0.010, P=0$. 013 for bootstrap), and correlated with poorer best drug response. Moreover, a possible correlation of CCR7 high expression and patients' baseline and post-administration lymph node metastasis was found.
\end{abstract}

Conclusions: High tumoral CCR7 expression correlated with potential lymphatic involvement and poor prognosis of mRCC patients treated with tyrosine kinase inhibitors. Further external validations and basic researches were needed to confirm these results.

Keywords: Metastatic renal cell carcinoma, CC-chemokine receptor 7, Overall survival, Progression free survival, Lymphatic invasion

\section{Background}

For patients with metastatic renal cell carcinoma (mRCC), therapeutic options have expanded significantly these years, since vascular endothelial growth factor (VEGF)-targeted tyrosine kinase inhibitor (TKI) drugs

\footnotetext{
* Correspondence: jjxufdu@fudan.edu.cn; guo.jianming@zs-hospital.sh.cn ${ }^{\dagger}$ Equal contributors

${ }^{2}$ Department of Biochemistry and Molecular Biology, School of Basic Medical Sciences, Fudan University, Mailbox 103138 Yixueyuan Road, Shanghai 200032, China

'Department of Urology, Zhongshan Hospital, Fudan University, 180 Fenglin Road, Shanghai 200032, China
}

such as sunitinib and pazopanib have been established as first-line therapy [1]. Several clinical based prognostic models, for example the Heng's risk criteria, have also achieved remarkable progress in mRCC patient survival prediction [2]. However, the objective response rates (ORRs) for most first-line TKI drugs were only around $30 \%$ [3], and the lack of validated molecular biomarkers impeded their personalized approach [4]. This was in contrast to many other tumor types, in which protein expression and mutation were used as basic accesses for drugs response and patient survival prediction $[5,6]$. 
CC-chemokine receptor 7 (CCR7), a G-protein coupled receptor (GPCR) mostly expressed on immune cells, was initially regarded as an important regulator facilitating leukocytes homing to the lymphatic structures, where its two ligands CC-chemokine ligand 19 (CCL19) and CCL21 are constitutively expressed [7]. However, in recent years, aberrant high CCR7 expression has also been identified on several tumor types, linking to a potential invasive phenotype [8]. It has been suggested that CCR7 positive tumor cells could mimic the normal lymphocyte homing function and interact with lymph vessels, leading to subsequent lymph node specific metastasis [9].

Lymphatic and hematogenous disseminations were two regular metastasis pathways for malignancy. For mRCC, although the most common metastatic site was the lung, possibly via a hematogenous approach, local or distant lymph node involvement at diagnosis was not rare [10]. Patient receiving TKIs could also develop new lymph node metastasis during the treatment, leading to a progressive disease (PD). Several theories of TKI drug resistance emphasized an increase of tumor cell invasiveness after drug administration [11]. These processes mostly accompanied with tumor cell migration and matrix metalloproteinase-9 (MMP-9) mediated extracellular matrix degradation [12], which was similar to the CCR7 mediated lymph vessel intravasation process [8]. As VEGF targeted therapies mostly focused on blood vessels inhibition, whether they could enhance the possibility of mRCC metastasis through other pathways, such as CCR7 mediated lymph vessel invasion and therapy resistance, was not known.

Thus, here through immunohistochemistry (IHC), we retrospectively evaluated the CCR7 expression in 110 primary tumor specimens of $\mathrm{mRCC}$ patients treated with sunitinib and sorafenib. The result suggested a positive correlation of CCR7 expression with patient baseline lymph node metastasis and TKI drugs response. CCR7 high expression could predict a poorer overall survival (OS) and progression free survival (PFS) for mRCC patients after TKIs.

\section{Methods}

\section{Patient selection}

We initially screened a total of 138 mRCC patients treated with sunitinib or sorafenib between March 2005 and June 2014 at the Department of Urology, Zhongshan Hospital, Fudan University. The inclusion criteria were: pathologically proven RCC patient with metastatic lesion, treated with sunitinib or sorafenib at first without further second-line treatment, had enough Formalin Fixed Paraffin Embedded (FFPE) specimens, and had detailed laboratorial, imaging and survival information. Patients who had former malignant history, received metastasectomy or those with tumor necrosis area
$>80 \%$, unavailable data were excluded. At last, 28 patients were excluded and 110 patients were selected for the study, in which three were excluded from PFS analysis for incomplete drug response information. This study was approved by the Clinical Research Ethics Committee of Zhongshan Hospital, Fudan University (Shanghai, China) (B2015-030).

Patients' OS was defined as the time from therapy initiation to the time of death, or was censored at the last follow-up. PFS was calculated from the time of therapy initiation to the time of progression, according to the RECIST 1.1 criteria [13], or was censored at the last follow-up. All data were collected retrospectively from medical records and electronic databases using uniform database templates, and the last follow-up time was December 2015. According to the 2014 EAU guidelines [14] and 2012 ISUP consensus [15], two urologic pathologists (Yuan J. and Jun H.) independently reviewed all the $\mathrm{H} \& \mathrm{E}$ slides of patient samples and confirmed the RCC diagnosis and Fuhrman grade classification. Initial stage at diagnosis was reclassified based on the 2010 AJCC TNM classification [10]. Heng's risk model was applied as previously reported [2].

\section{Tissue microarray and immunohistochemistry}

Two representative tumor cores $3 \mathrm{~mm}$ in diameter from each sample were selected for tissue micro array (TMA) construction. Anti-CCR7 antibody (ab32527, Abcam, diluted 1/1000) and Dako EnVision Detection System were applied in the immunohistochemistry procedure [16]. Through western blot in RCC cell lines, the specificity of antibody was confirmed. Negative control was performed without applying primary antibody. Olympus CDD camera, Nikon eclipse Ti-s microscope $(\times 200$ magnification) and NIS-Elements F3.2 software were used to record the staining results. Using Image-Pro Plus version 6.0 software (Media Cybernetics Inc., USA), an integrated optical density (IOD) score could be calculated for each scan. Two urologists unaware of the patients' clinical data evaluated these slides. Each person took three independent shots with the strongest staining for each core, and the IOD mean of each patient's two cores (six scans) were calculated. Kappa value was calculated for evaluating inter-observer agreement.

\section{Statistical analysis}

Univariate analysis was carried out to explore the prognostic and predictive value of continuous CCR7 IOD score. The smooth estimates of hazard ratio (HR) of IOD on patient survival were displayed using R software, "phenoTest" package [17]. For clinical usage, we dichotomized the IOD into high/low expression through minimum $\mathrm{p}$ value method using $\mathrm{x}$-tile software [18], and because the $p$ values obtained might be overestimated, 
Table 1 Clinical characteristics of patients according to tumoral CCR7 expression

\begin{tabular}{|c|c|c|c|c|c|}
\hline \multirow[t]{2}{*}{ Characteristics } & \multicolumn{2}{|c|}{ Patients } & \multicolumn{3}{|c|}{ Tumoral CCR7 expression } \\
\hline & $\bar{n}$ & $\%$ & low & high & $P$-value \\
\hline No. of patients & 110 & 100 & 53 & 57 & \\
\hline Age, years, mean (SD) & \multicolumn{2}{|c|}{$57.5(11.9)$} & & & \\
\hline Men & 79 & 71.8 & & & \\
\hline ECOG PS & & & & & $0.198^{c}$ \\
\hline 0 & 81 & 73.6 & 42 & 39 & \\
\hline 1 & 29 & 26.4 & 11 & 18 & \\
\hline Prior nephrectomy & & & & & N/A \\
\hline Yes & 110 & 100.0 & & & \\
\hline No & 0 & 0.0 & & & \\
\hline Histology & & & & & $0.086^{c}$ \\
\hline Clear cell & 88 & 80.0 & 46 & 42 & \\
\hline Non-clear cell type & 22 & 20.0 & 7 & 15 & \\
\hline Papillary & 15 & 13.6 & & & \\
\hline Chromophobe & 2 & 1.8 & & & \\
\hline Collecting duct & 2 & 1.8 & & & \\
\hline Unclassified & 3 & 2.7 & & & \\
\hline Fuhrman grade (7 excluded) & & & & & $0.147^{d}$ \\
\hline 1 & 2 & 1.8 & 2 & 0 & \\
\hline 2 & 53 & 48.2 & 28 & 25 & \\
\hline 3 & 41 & 37.3 & 18 & 23 & \\
\hline 4 & 7 & 6.4 & 5 & 2 & \\
\hline TNM stage at initial diagnosis & & & & & $0.004^{c}$ \\
\hline$|-|||$ & 51 & 46.4 & 32 & 19 & \\
\hline IV & 59 & 53.6 & 21 & 38 & \\
\hline Site of metastatic disease ${ }^{a}$ & & & & & \\
\hline Lung & 82 & 74.5 & & & \\
\hline Liver & 13 & 11.8 & & & \\
\hline Bone & 18 & 16.4 & & & \\
\hline Lymph node & 29 & 26.4 & & & \\
\hline Adrenal gland & 8 & 7.3 & & & \\
\hline Brain and others & 15 & 13.6 & & & \\
\hline Lymph node involvement ${ }^{a}$ & & & & & $0.031^{c}$ \\
\hline No & 81 & 73.6 & 44 & 37 & \\
\hline Yes & 29 & 26.4 & 9 & 20 & \\
\hline No. of metastatic sites ${ }^{a}$ & & & & & $0.070^{c}$ \\
\hline 1 & 76 & 69.1 & 41 & 35 & \\
\hline$\geq 2$ & 34 & 30.9 & 12 & 22 & \\
\hline Tyrosine kinase inhibitors & & & & & $0.254^{c}$ \\
\hline Sunitinib & 73 & 66.4 & 38 & 35 & \\
\hline Sorafenib & 37 & 33.6 & 15 & 22 & \\
\hline Heng's risk group & & & & & $0.271^{d}$ \\
\hline
\end{tabular}

Table 1 Clinical characteristics of patients according to tumoral CCR7 expression (Continued)

\begin{tabular}{|c|c|c|c|c|c|}
\hline Favorable & 22 & 20 & 12 & 10 & \\
\hline Intermediate & 60 & 54.5 & 30 & 30 & \\
\hline Poor & 28 & 25.5 & 11 & 17 & \\
\hline Best response (3 not assessable) & & & & & $<0.001^{d}$ \\
\hline Partial response & 27 & 25.2 & 18 & 9 & \\
\hline Stable disease for $\geq 3$ months & 57 & 53.3 & 30 & 27 & \\
\hline Progressive disease $^{b}$ & 23 & 21.5 & 2 & 21 & \\
\hline \multicolumn{6}{|c|}{$\begin{array}{l}\text { Bold data means statistical significant }(P<0.05) \\
S D \text { standard deviation, ECOG PS Eastern Cooperative Oncology Group } \\
\text { performance status } \\
P \text {-value }<0.05 \text { was regarded as statistically significant } \\
{ }^{a} \text { At the time initializing tyrosine kinase inhibitors } \\
{ }^{b} \text { Including stable disease for }<3 \text { months } \\
{ }^{c} X^{2} \text { test or Fisher's exact test } \\
{ }^{d} \text { Cochran-Mantel-Haenszel } X^{2} \text { test }\end{array}$} \\
\hline
\end{tabular}

they were corrected using the formula proposed by Altman and colleagues [19]. The smooth HR curves after dichotomizing were illustrated through $\mathrm{R}$ software, "smoothHR" package [20]. After this, $\chi 2$ test, Fisher's exact method and Cochran-Mantel-Haenszel $\chi 2$ test were applied for assessing the relationship between CCR7 expression and patients' clinicopathological parameters. Kaplan-Meier, univariate and subsequent multivariate analysis were performed, in which 1000 bootstrap resamples was used for reducing overfitting bias. Finally, time dependent receiver operating characteristic (ROC) analysis was done to analyze the adding prognostic value of CCR7 expression to the Heng's risk model. GraphPad Prism 6 (GraphPad Software Inc., USA), SPSS 21.0 (SPSS Inc., USA), X-tile 3.6.1 (Robert L Camp, USA) and $\mathrm{R}$ software 3.1.2 (R Foundation for Statistical Computing, Austria) were used in these procedures. A two-sided P-value $<0.05$ was regarded as statistically significant.

\section{Results}

CCR7 staining and cut off point choosing

CCR7 expression in the RCC sample was variable, and mostly on the membrane and cytoplasm of tumor cells (Additional file 1: Figure S1A and B). Its expression in peritumoral tissue was relatively low (Additional file 1: Figure S1C). Inter-observer agreement was acceptable according to the kappa value 0.745 , thus the CCR7 IOD means from the two observers were again averaged as the final IOD, and the distributions were 73-584 for range; $238 \pm 97$ for mean and SD; 223 (173-287) for median and IQR.

In order to select an appropriate cut off value for clinical usage, first we performed a univariate analysis using the continuous CCR7 IOD score (Additional file 2: Table S1). Result suggested CCR7 as a significant adverse prognostic marker for mRCC patients' OS and PFS $(P<0.001$ for 
both). Smooth HR curve demonstrated the adding risk of 1 per IOD on patient survival (Additional file 1: Figure S1D and F). Then, through minimum $p$ value method using log rank test, IOD $=215$ was chosen as the cut off point $(P<0.001$ for both), and the $\mathrm{p}$ values were still significant after being corrected (OS, $P<0.001$, PFS, $P=$ 0.001). The smooth HR curve also displayed significant and stable prognostic differences after dichotomizing (Additional file 1: Figure S1E and G).

\section{Patient baseline characteristics and its association with dichotomous CCR7 expression}

The 110 patients' baseline clinical characteristics were shown in Table 1. All patients were East Asian. The medium age at TKIs initiation was 59 years old (range 14-78). All patients have received radical, partial or cytoreductive nephrectomy. Results showed that the dichotomous CCR7 expression associated with TNM stage at initial diagnosis $(P=0.004)$, lymph node involvement $(P=0.031)$ and marginally with histology $(P=0.086)$ and number of metastatic sites $(P=0.070)$. It also correlated with patients' best response of TKIs $(P<0.001)$.

Impact of baseline characteristics, including dichotomous CCR7 expression, on OS in mRCC patients receiving TKIs

Within this cohort, 64.5\% (71/110) patients died during the follow-up and the median OS was 23.5 months. Kaplan-Meier analysis revealed that patients with high CCR7 expression had a significantly poorer OS $(P<0.001$ after correction) (Fig. 1a). Univariate analysis confirmed this significance $(P<0.001)$ (Additional file 2: Table S1). Multivariate Cox analysis was further performed and suggested high CCR7 expression as an independent adverse prognostic factor for mRCC patients' OS prediction (HR 2.256, 95\% CI
1.336-3.809, $P=0.002 ; P=0.003$ after 1000 bootstrap), together with tumor histology and Heng's risk group (Table 2). Lung and lymph node involvement were excluded from the model for being potential confounding factors for number of metastatic sites.

Stratified analysis were further performed, and we found that CCR7 expression could discriminate most patient groups' overall survival except those in the nonclear cell type or Heng's risk favorable/poor groups (Additional file 3: Table S2). But after incorporating the high/low CCR7 expression directly into Heng's risk to form a new model, the OS between different groups displayed vigorously discriminative consequences $(P<0.001)$ (Fig. 1b). Furthermore, ROC analysis was carried out at the time of 12 and 24-month follow-up, and the new model showed better prognostic power than using Heng's risk model alone in both the ccRCC and all patient groups (Fig. 2). Incorporating CCR7 IOD score as a continuous variable also displayed similar results (Additional file 4: Figure S2).

Impact of baseline characteristics, including dichotomous CCR7 expression, on PFS in mRCC patients receiving TKIs

During the follow-up period, 85.0\% (91/107) patients have developed disease progression. The median PFS was 9.8 months. Patients' best response and its correlation with CCR7 were shown in Table 1. Figure 3a revealed that RCC in the PD group displayed a significantly higher CCR7 expression compared to partial response (PR) and stable disease (SD) groups. Kaplan-Meier analysis suggested an adverse predictive effect of high CCR7 expression in patients receiving TKIs $(P=0.001$ after correction) (Fig. 3b) and was also confirmed in a multivariate model (HR 1.835, 95\% CI 1.156-2.912, $P=0.010 ; P=0.013$ after 1000 bootstrap) (Table 2). After incorporating CCR7 into the Heng's model, patients in the new model
A

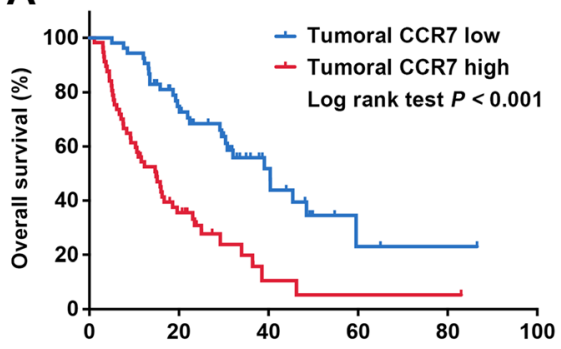

No. at risk Time after therapy initiation (months) Tumoral CCR7 low

\begin{tabular}{cccccc}
53 & 36 & 13 & 2 & 1 & 0 \\
\multicolumn{2}{l}{ Tumoral CCR7 high } & & & & \\
57 & 18 & 2 & 1 & 1 & 0
\end{tabular}

B

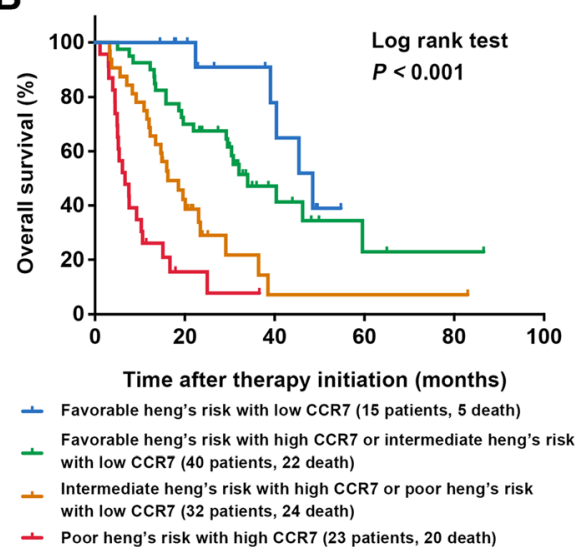

Fig. 1 Impact of tumoral CCR7 expression on patients' overall survival (OS). a OS according to tumoral CCR7 expression; b Heng's risk model expanded with tumoral CCR7 expression 


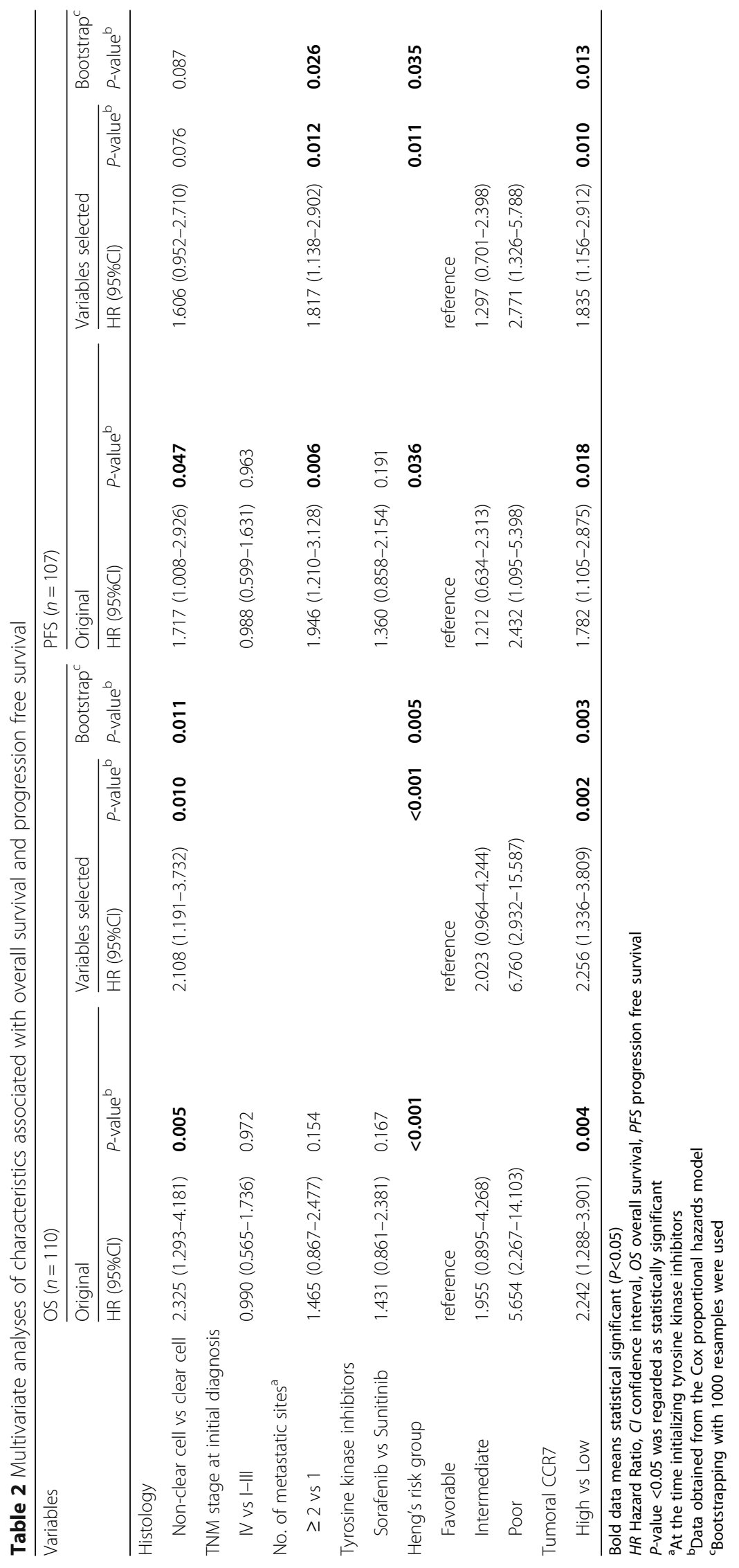


A
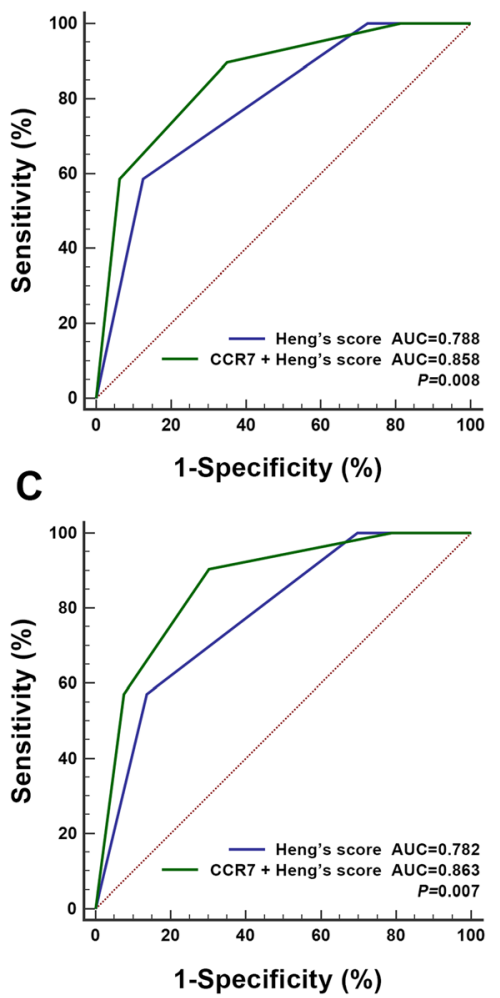

B
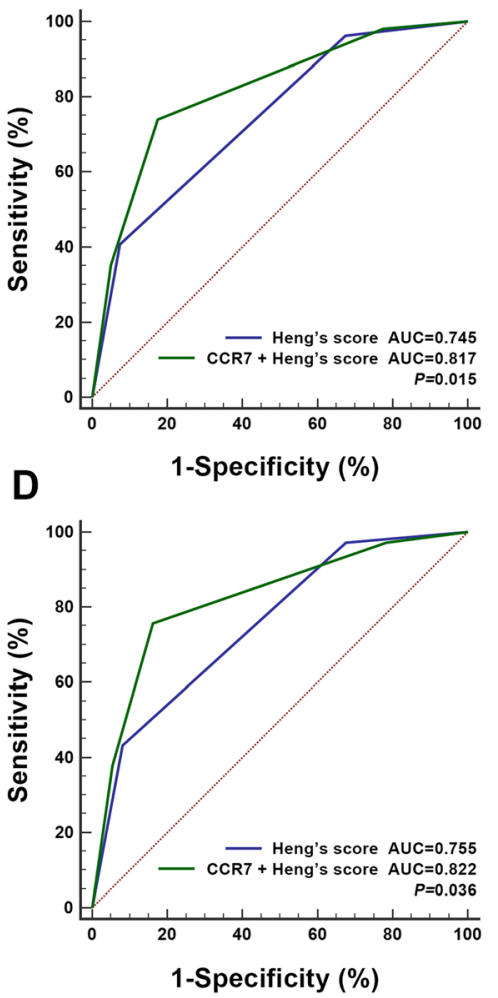

Fig. 2 ROC analysis of Heng's risk model alone and expanded with CCR7 expression on patients' OS. a all patients at 12 months; $\mathbf{b}$ all patients at 24 months; c pathologic clear cell type at 12 months; $\mathbf{d}$ pathologic clear cell type at 24 months

displayed significant PFS divergence between different groups $(P<0.001)$ (Fig. 3c). Since the Heng's risk criteria was initially designed for OS prediction, further ROC comparison was not performed.

\section{CCR7 expression and its correlation with lymph node involvement}

In Fig. 4a, the CCR7 IOD score of mRCC patients with different baseline metastatic sites were plotted, and revealed a potential higher expression of CCR7 in patients with baseline lymph node metastasis, in accordance with the $\chi 2$ test in Table 1, though the Kruskal-Wallis test did not meet statistical significance $(P=0.083)$. For exploring the possible impact of CCR7 on lymphatic invasion during the drug treatment period, we found that four patients within this cohort have developed disease progression due to new lymph node lesions development, and all their tumor samples displayed CCR7 high expression (Fig. 4b).

\section{Discussion}

CCR7 was naturally a homeostatic chemokine receptor expressed on various subtypes of immune cells encompassing $\mathrm{T}$ cells, $\mathrm{B}$ cells, natural killer cells and dendritic cells, enabling them to circulate through the CCL-19/21 positive lymphatic highways [7]. Its expression on cancer cells was first recognized on hematogenous malignancies, in which the connection between high CCR7 expression and lymphoid organ involvement was discovered [21]. Subsequently, numerous laboratory studies confirmed this pro-invasion, mostly pro-lymph vessel metastatic function in various cancer types, including breast cancer, melanoma, non-small cell lung cancer, prostate cancer, headand-neck cancer and gastrointestinal cancer [9]. Here, our results suggested that CCR7 also expressed on several RCC specimens and associated with various patient baseline characteristics (Table 1, Additional file 1: Figure S1).

The clinical prognostic value of CCR7 has been studied in several other cancer types. In a meta-analysis including 1697 gastric cancer patients, high CCR7 correlated with a worse 5-year overall survival rate [22]. Studies in melanoma and colorectal cancer also displayed similar results [23, 24]. In this study, we have found that high CCR7 staining intensity could be used as an adverse prognosticator for mRCC patients' overall survival. Moreover, a new model integrating CCR7 expression into Heng's risk criteria performed better than using Heng's risk alone, and the adding prognostic value mostly came from the intermediate patient groups (Additional file 3: Table S2, Fig. 2a and b). This indicated 


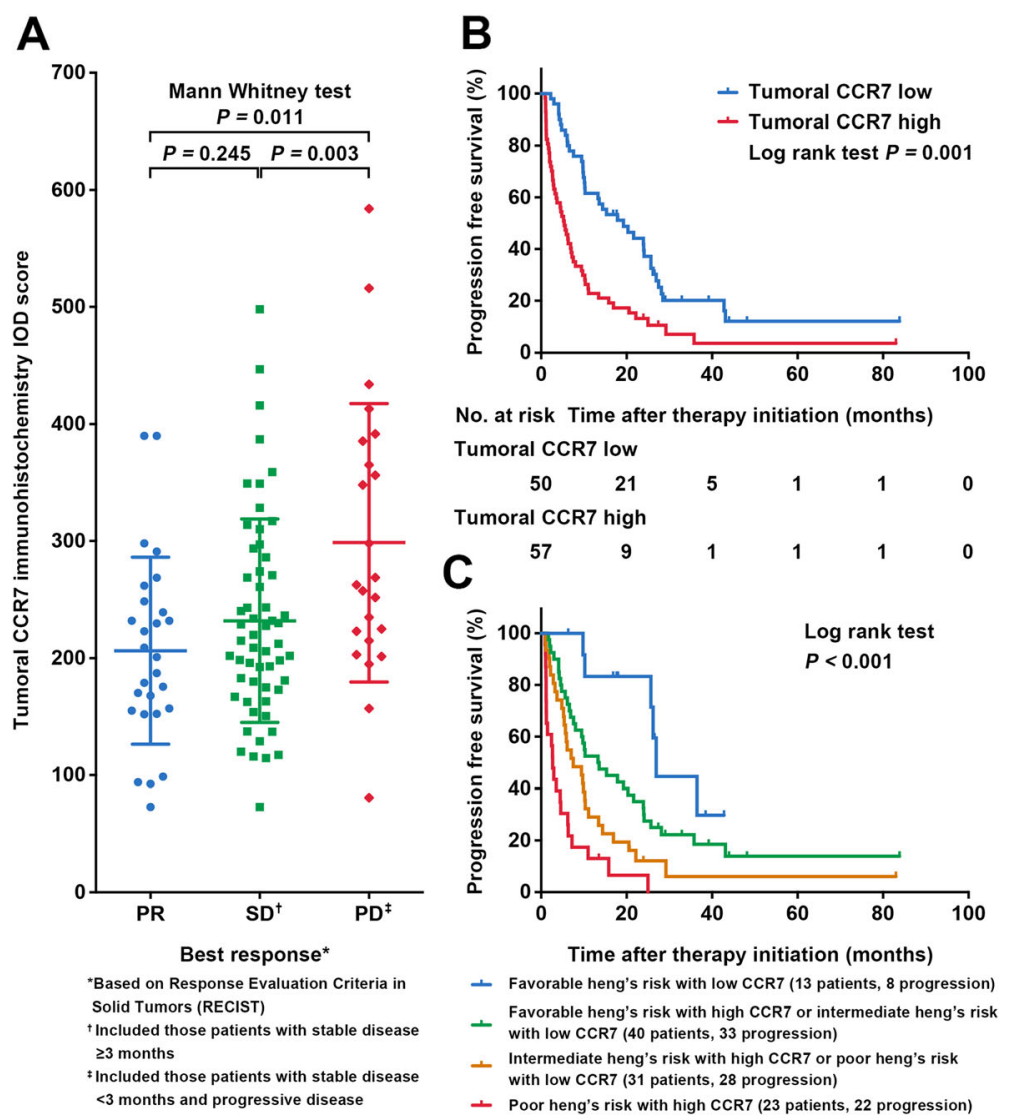

Fig. 3 Impact of tumoral CCR7 expression on patients' best drug response for tyrosine kinase inhibitors and PFS. a Patients' best drug response according to tumoral CCR7 expression; b PFS according to tumoral CCR7 expression; $\mathbf{c}$ Heng's risk model expanded with tumoral CCR7 expression

that a substantial number of patients might switch between risk groups with a consequence for choice of treatment strategy after CCR7 status was considered. ROC analysis for the ccRCC patient group were also carried out, considering the poor performance of CCR7 in non-ccRCC patients (Fig. 2c and d).

For PFS analysis, to our knowledge, this study was the first to report an association between tumoral CCR7 expression and PFS in mRCC patients following TKIs. As the most widely used systemic therapy at present, TKI drugs only reached ORRs for about $30 \%$ for mRCC patients, and the theories of drug resistance was complicated [25]. It is increasingly evident that in some tumors, in which angiogenesis is thwarted genetically or pharmacologically, cancer cells could adapt by migrating more aggressively into normal tissue, based on several pre-existing invasion programmes such as epithelial-mesenchymal transition (EMT) and MMP2/MMP9 secretion [26, 27], or by switching on several distinctive programmes which were currently unknown [28]. Since the CCR7 mediated lymphatic specific migration and metastasis was also based on several above mentioned programmes [8, 29], and as a molecule which could give tumor itself survival signal besides [30], we hypothesized that CCR7 positive RCC cells might have a potential to migrate into the adjacent lymphatic tissue for survival after TKI drugs administration, subsequently leading to resistance and disease progression. As a result, our study did identify an independent significant adverse predictive effect of high CCR7 expression on mRCC patients' PFS (Fig. 3b) and its association with poorer best drug response (Table 1, Fig. 3a). Patients' baseline lymph node involvement status was also correlated (Table 1, Fig. 4a) and four patients who developed PD due to new lymph node metastasis all represented high CCR7 staining for their tumor tissues. All these results above indicated an impact of tumoral CCR7 expression on patients' PFS and lymphatic involvement status, making this molecule a potential predictor for mRCC patients receiving TKIs.

The major limitations of this pilot study were its retrospective design and relatively small sample size, with patients from a single center and same ethnic background. Although central patient data review and bootstrap validation were performed for minimizing inter-observer and over-fitting bias, further external prospective validations were required, and related basic researches were 

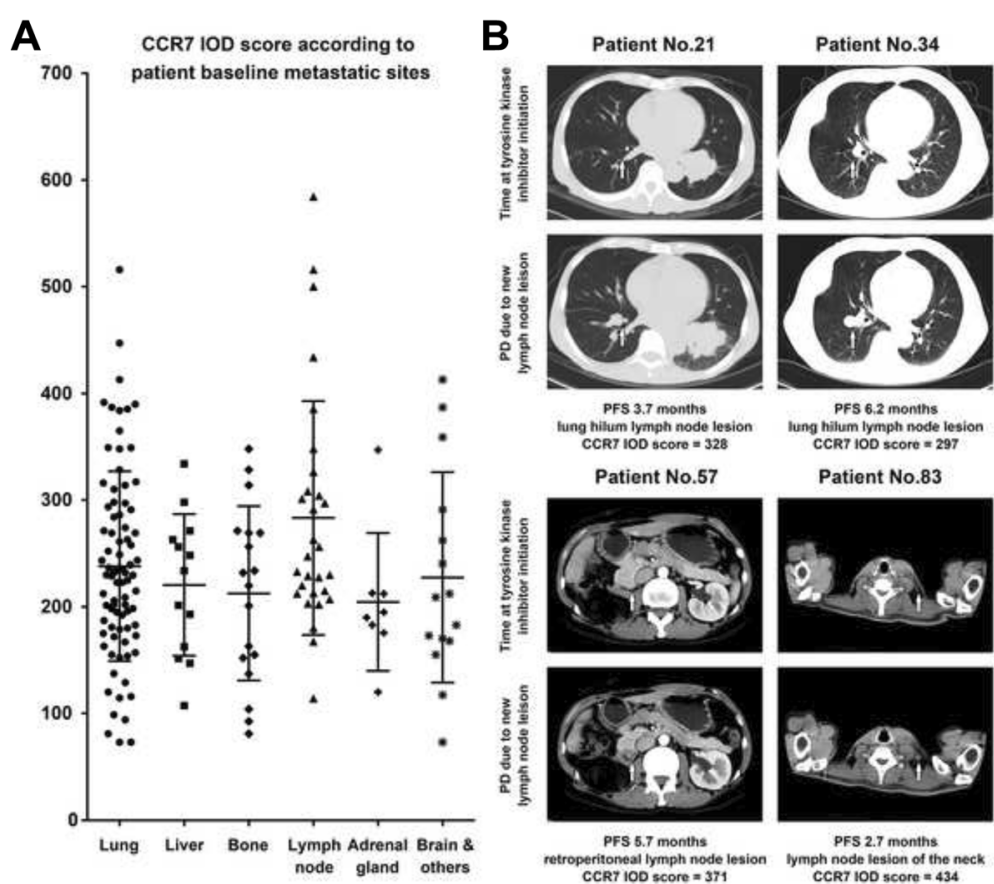

Fig. 4 Correlation of tumoral CCR7 expression and patients' baseline and post-administration lymphatic involvements. a Tumoral CCR7 expression according to different patient baseline metastatic sites; b Four mRCC patients who have experienced disease progression due to new lymphatic lesions development after tyrosine kinase inhibitors, all with high CCR7 expression. White arrow: the area where new lymph node lesions developed during administration

needed for verifying our hypothesis. Samples from the metastatic site after TKI therapies might be excellent candidates for in-depth investigation. Besides, although we took two cores and six scans from each patient's sample, intratumoral heterogeneity still might confound the results. Patients in this study received sunitinib or sorafenib as first-line therapy because other agents were not available in China at that time, and other VEGFbased TKI drugs such as pazopanib, axitinib and cabozantinib should also be taken into further consideration.

\section{Conclusions}

Our study indicated that high tumoral CCR7 expression correlated with potential lymphatic involvement and poor prognosis in mRCC patients treated with TKIs. This biomarker could also be combined with the Heng's risk model for better risk stratification.

\section{Additional files}

Additional file 1: Figure S1. Representative photographs of CCR7 immunostaining and cut-off point choosing. (A) Tumoral CCR7 low expression; (B) Tumoral CCR7 high expression; (C) Peritumoral CCR7 expression. Original magnification $\times 200$; (D), (F) Smooth estimates of HR (+1 IOD) showed a higher risk of death and progression for patients with stronger tumoral CCR7 staining; (E), (G) Smooth estimates of HR (using $\mathrm{IOD}=215$ as a reference) showed a significant and stable prognostic difference between patients with high/low tumoral CCR7 staining. Dashed lines: 95\% confidence bands. (TIF $5423 \mathrm{~kb}$ )

Additional file 2: Table S1. Univariate analyses of characteristics associated with overall survival and progression free survival. (DOCX $69 \mathrm{~kb}$ )

Additional file 3: Table S2. Hazard ratios for OS and PFS based on CCR7 in different subgroups (High vs Low). (DOCX 63 kb)

Additional file 4: Figure S2.ROC analysis of Heng's risk model alone and expanded with CCR7 continuous IOD score on patients' OS. (A) all patients at 12 months; (B) all patients at 24 months; (C) pathologic clear cell type at 12 months; (D) pathologic clear cell type at 24 months. (TIF $1371 \mathrm{~kb}$ )

\section{Abbreviations}

CCR7: CC-chemokine receptor 7; ccRCC: Clear-cell renal cell carcinoma; HR: Hazard ratio; IHC: Immunohistochemistry; IOD: Integrated optical density; mRCC: Metastatic renal cell carcinoma; ORR: Objective response rate; OS: Overall survival; PD: Progressive disease; PFS: Progression free survival; RCC: Renal cell carcinoma; ROC: Receiver operating characteristic; TKl: Tyrosine kinase inhibitor

\section{Acknowledgments}

The authors would like to thank Dr. Yuan Ji, Dr. Jun Hou and Ms. Haiying Zeng (Department of Pathology, Zhongshan Hospital of Fudan University) for diagnosis confirmation and technical assistance, respectively.

\section{Funding}

This study was funded by grants from National Key Projects for Infectious Diseases of China (2012ZX10002012-007, 2016ZX10002018-008), National Natural Science Foundation of China $(31100629,31270863,81372755,31470794$, $81402082,81402085,81471621,81472227,81472376,31570803,81501999$, 81671628 and 81672324), Program for New Century Excellent Talents in University (NCET-13-0146) and Science and Technology Commission of Shanghai Municipality (14ZR1406300). All these study sponsors have no roles in the study design, in the collection, analysis and interpretation of data. 


\section{Availability of data and materials}

The basic patient information and $\mathrm{HC}$ staining results have been shown in Table 1 and Additional file 1: Figure S1. And we are sorry that the detailed dataset supporting the conclusions could not be provided at present, because the cohort information was currently under an update. We are now adding more mRCC patients (diagnosed from 2014 to 2016) into the cohort and refreshing the follow up information (till 2016). Once this is done, we would like to combine CCR7 with other biomarkers for better mRCC patient survival prediction, and at that time those updated information would be available to the readers.

\section{Authors' contributions}

YX carried out and conducted experiments, performed statistical analysis and drafted the manuscript. LL participated in the collection of patient materials and drafting of the manuscript. YX carried out laboratory work and data analysis. JW and QB performed laboratory work and participated in the correction of words in the manuscript. WX and YQ participated in the study design and collection of related articles. JX took charge of the study design and revising manuscript critically for important intellectual content. JG conceived of the study, and led the data analysis and oversaw the drafting of the manuscript. All authors read and approved the final manuscript.

\section{Competing interests}

The authors declare that they have no competing interests.

\section{Consent for publication}

Not applicable.

\section{Ethics approval and consent to participate}

This study was approved by the Clinical Research Ethics Committee of Zhongshan Hospital, Fudan University (Shanghai, China) (B2015-030). Al specimens were obtained from patients with written informed consent.

Received: 25 August 2016 Accepted: 16 January 2017

Published online: 23 January 2017

\section{References}

1. Di Lorenzo G, Autorino R, Sternberg CN. Metastatic renal cell carcinoma: recent advances in the targeted therapy era. Eur Urol. 2009;56(6):959-71.

2. Heng DY, Xie W, Regan MM, Warren MA, Golshayan AR, Sahi C, Eigl BJ, Ruether JD, Cheng T, North S, et al. Prognostic factors for overall survival in patients with metastatic renal cell carcinoma treated with vascular endothelial growth factor-targeted agents: results from a large, multicenter study. J Clin Oncol Off J Am Soc Clin Oncol. 2009;27(34):5794-9.

3. Sternberg CN, Davis ID, Mardiak J, Szczylik C, Lee E, Wagstaff J, Barrios CH, Salman P, Gladkov OA, Kavina A, et al. Pazopanib in locally advanced or metastatic renal cell carcinoma: results of a randomized phase III trial. J Clin Oncol Off J Am Soc Clin Oncol. 2010;28(6):1061-8

4. Stewart GD, O'Mahony FC, Powles T, Riddick AC, Harrison DJ, Faratian D. What can molecular pathology contribute to the management of renal cell carcinoma? Nat Rev Urol. 2011;8(5):255-65.

5. Druker BJ, Guilhot F, O'Brien SG, Gathmann I, Kantarjian H, Gattermann N, Deininger MW, Silver RT, Goldman JM, Stone RM, et al. Five-year follow-up of patients receiving imatinib for chronic myeloid leukemia. N Engl J Med. 2006;355(23):2408-17.

6. Slamon D, Eiermann W, Robert N, Pienkowski T, Martin M, Press M, Mackey J, Glaspy J, Chan A, Pawlicki M, et al. Adjuvant trastuzumab in HER2-positive breast cancer. N Engl J Med. 2011;365(14):1273-83.

7. Forster R, Davalos-Misslitz AC, Rot A. CCR7 and its ligands: balancing immunity and tolerance. Nat Rev Immunol. 2008;8(5):362-71.

8. Legler DF, Uetz-von Allmen E, Hauser MA. CCR7: roles in cancer cell dissemination, migration and metastasis formation. Int J Biochem Cell Biol. 2014; $54: 78-82$

9. Zlotnik A, Burkhardt AM, Homey B. Homeostatic chemokine receptors and organ-specific metastasis. Nat Rev Immunol. 2011;11(9):597-606.

10. Kim SP, Alt AL, Weight CJ, Costello BA, Cheville JC, Lohse C, Allmer C, Leibovich BC. Independent validation of the 2010 American Joint Committee on Cancer TNM classification for renal cell carcinoma: results from a large, single institution cohort. J Urol. 2011;185(6):20359.

11. Loges $S$, Mazzone M, Hohensinner $P$, Carmeliet P. Silencing or fueling metastasis with VEGF inhibitors: antiangiogenesis revisited. Cancer Cell. 2009;15(3):167-70

12. Joosten SC, Hamming L, Soetekouw PM, Aarts MJ, Veeck J, van Engeland M, Tjan-Heijnen VC. Resistance to sunitinib in renal cell carcinoma: From molecular mechanisms to predictive markers and future perspectives. Biochim Biophys Acta. 2015;1855(1):1-16.

13. Eisenhauer EA, Therasse P, Bogaerts J, Schwartz LH, Sargent D, Ford R, Dancey J, Arbuck S, Gwyther S, Mooney M, et al. New response evaluation criteria in solid tumours: revised RECIST guideline (version 1.1). Eur J Cancer. 2009;45(2):228-47. Oxford, England: 1990.

14. Ljungberg B, Bensalah K, Canfield S, Dabestani S, Hofmann F, Hora M, Kuczyk MA, Lam T, Marconi L, Merseburger AS, et al. EAU guidelines on renal cell carcinoma: 2014 update. Eur Urol. 2015;67(5):913-24.

15. Delahunt B, Cheville JC, Martignoni G, Humphrey PA, Magi-Galluzzi C, McKenney J, Egevad L, Algaba F, Moch H, Grignon DJ, et al. The International Society of Urological Pathology (ISUP) grading system for renal cell carcinoma and other prognostic parameters. Am J Surg Pathol. 2013; 37(10):1490-504.

16. Pan D, Xu L, Liu H, Zhang W, Zhu Y, Xu J, Gu J. Interleukin-11 receptor predicts post-operative clinical outcome in patients with early-stage clearcell renal cell carcinoma. Jpn J Clin Oncol. 2015;45(2):202-9.

17. Calon A, Lonardo E, Berenguer-Llergo A, Espinet E, Hernando-Momblona X Iglesias M, Sevillano M, Palomo-Ponce S, Tauriello DV, Byrom D, et al. Stromal gene expression defines poor-prognosis subtypes in colorectal cancer. Nat Genet. 2015;47(4):320-9.

18. Camp RL, Dolled-Filhart M, Rimm DL. X-tile: a new bio-informatics tool for biomarker assessment and outcome-based cut-point optimization. Clin Cancer Res. 2004;10(21):7252-9.

19. Altman DG, Lausen B, Sauerbrei W, Schumacher M. Dangers of using "optimal" cutpoints in the evaluation of prognostic factors. J Natl Cancer Inst. 1994;86(11):829-35.

20. Meira-Machado L, Cadarso-Suarez C, Gude F, Araujo A. smoothHR: an $R$ package for pointwise nonparametric estimation of hazard ratio curves of continuous predictors. Comput Math Methods Med. 2013; 2013:745742.

21. Hasegawa H, Nomura T, Kohno M, Tateishi N, Suzuki Y, Maeda N, Fujisawa R, Yoshie O, Fujita S. Increased chemokine receptor CCR7/EBI1 expression enhances the infiltration of lymphoid organs by adult T-cell leukemia cells. Blood. 2000:95(1):30-8

22. Du P, Liu Y, Ren H, Zhao J, Zhang X, Patel R, Hu C, Gan J, Huang G. Expression of chemokine receptor CCR7 is a negative prognostic factor for patients with gastric cancer: a meta-analysis. Gastric Cancer. 2016;1-11. doi:10.1007/s10120-016-0602-8.

23. Malietzis G, Lee GH, Bernardo D, Blakemore Al, Knight SC, Moorghen M, AlHassi HO, Jenkins JT. The prognostic significance and relationship with body composition of CCR7-positive cells in colorectal cancer. J Surg Oncol. 2015; 112(1):86-92.

24. Kuhnelt-Leddihn L, Muller H, Eisendle K, Zelger B, Weinlich G. Overexpression of the chemokine receptors CXCR4, CCR7, CCR9, and CCR10 in human primary cutaneous melanoma: a potential prognostic value for CCR7 and CCR10? Arch Dermatol Res. 2012; 304(3):185-93.

25. Bergers G, Hanahan D. Modes of resistance to anti-angiogenic therapy. Nat Rev Cancer. 2008:8(8):592-603.

26. Hammers HJ, Verheul HM, Salumbides B, Sharma R, Rudek M, Jaspers J, Shah $\mathrm{P}$, Ellis L, Shen L, Paesante $\mathrm{S}$, et al. Reversible epithelial to mesenchymal transition and acquired resistance to sunitinib in patients with renal cell carcinoma: evidence from a xenograft study. Mol Cancer Ther. 2010;9(6):1525-35.

27. Perez-Gracia JL, Prior C, Guillen-Grima F, Segura V, Gonzalez A, Panizo A, Melero I, Grande-Pulido E, Gurpide A, Gil-Bazo I, et al. Identification of TNFalpha and MMP-9 as potential baseline predictive serum markers of sunitinib activity in patients with renal cell carcinoma using a human cytokine array. Br J Cancer. 2009;101(11):1876-83.

28. Kunkel P, Ulbricht U, Bohlen P, Brockmann MA, Fillbrandt R, Stavrou D, Westphal M, Lamszus K. Inhibition of glioma angiogenesis and growth in vivo by systemic treatment with a monoclonal antibody against vascular endothelial growth factor receptor-2. Cancer Res. 2001;61(18):6624-8. 
29. Pang MF, Georgoudaki AM, Lambut L, Johansson J, Tabor V, Hagikura K, Jin Y, Jansson M, Alexander JS, Nelson CM, et al. TGF-beta1-induced EMT promotes targeted migration of breast cancer cells through the lymphatic system by the activation of CCR7/CCL21-mediated chemotaxis. Oncogene. 2016;35(6):748-60.

30. Mburu YK, Wang J, Wood MA, Walker WH, Ferris RL. CCR7 mediates

inflammation-associated tumor progression. Immunol Res. 2006;36(1-3):61-72.

Submit your next manuscript to BioMed Central and we will help you at every step:

- We accept pre-submission inquiries

- Our selector tool helps you to find the most relevant journal

- We provide round the clock customer support

- Convenient online submission

- Thorough peer review

- Inclusion in PubMed and all major indexing services

- Maximum visibility for your research

Submit your manuscript at www.biomedcentral.com/submit 